PROCEEDINGS OF THE

AMERICAN MATHEMATICAL SOCIETY

Volume 137, Number 8, August 2009, Pages 2655-2659

S 0002-9939(09)09893-1

Article electronically published on March 30, 2009

\title{
A NOTE ON CLASSIFICATION OF SUBMODULES IN $H^{2}\left(D^{2}\right)$
}

\author{
RONGWEI YANG
}

(Communicated by Nigel J. Kalton)

\begin{abstract}
The Hardy spaces $H^{2}\left(D^{2}\right)$ can be viewed as a module over the polynomial ring $C\left[z_{1}, z_{2}\right]$. Based on a study of core operator, a new equivalence relation for submodules, namely congruence, was introduced. This paper gives a classification of congruent submodules by the rank of core operators.
\end{abstract}

\section{INTRODUCTION}

In this paper $D$ denotes the unit disk of the complex plane $\mathbf{C}$ and $T$ denotes the unit circle. The polynomial ring $C\left[z_{1}, z_{2}\right]$ acts on the Hardy space over the bidisk $H^{2}\left(D^{2}\right)$ by multiplication of functions, which turns $H^{2}\left(D^{2}\right)$ into a module over $C\left[z_{1}, z_{2}\right]$. It is clear that a closed subspace $M \subset H^{2}\left(D^{2}\right)$ is a submodule if and only if it is invariant under multiplications by both $z_{1}$ and $z_{2}$. For example, if $I$ is an ideal in $C\left[z_{1}, z_{2}\right]$, then its closure in $H^{2}\left(D^{2}\right)$ (which we denote by $[I]$ ) is a submodule. There are also many submodules that are unrelated to ideals in $C\left[z_{1}, z_{2}\right]$. For instance, W. Rudin displayed two submodules in $\mathrm{Ru}$ : one is of infinite rank, and the other contains no nontrivial bounded functions. In an attempt to understand the structure of submodules, two canonical equivalence relations were considered. Two submodules $M$ and $N$ are said to be unitarily equivalent (or similar) if there is a unitary (or, respectively, invertible) module map between them. Much is known about the two equivalence relations (cf. Chen and Guo [CG]). A most notable fact is the rigidity phenomenon discovered by Douglas, Paulsen, Sah and Yan in [DPSY]. To be precise, let $I_{1}$ and $I_{2}$ be two ideals in $C\left[z_{1}, z_{2}\right]$ such that each has at most countably many zeros in $D^{2}$. If there are bounded module maps $A:\left[I_{1}\right] \longrightarrow\left[I_{2}\right]$ and $B:\left[I_{2}\right] \longrightarrow\left[I_{1}\right]$ both with dense range, then $\left[I_{1}\right]=\left[I_{2}\right]$. Hence $\left[I_{1}\right]$ and $\left[I_{2}\right]$ are unitarily equivalent or similar only if they are identical. The following example provides a simple illustration of this fact.

Example 1. Let $\lambda=\left(\lambda_{1}, \lambda_{2}\right)$ be any point in $D^{2}$ and

$$
H_{\lambda}=\left\{f \in H^{2}\left(D^{2}\right): f(\lambda)=0\right\} .
$$

Then $H_{\lambda}$ is a submodule. The rigidity theorem above implies that as long as $\alpha \neq \beta$, $H_{\alpha}$ and $H_{\beta}$ are not unitarily equivalent.

Received by the editors September 9, 2008.

2000 Mathematics Subject Classification. Primary 47A13; Secondary 46E20.

Key words and phrases. Core operator, congruence, Hardy space, submodules.

This work is supported in part by a grant from the National Science Foundation (DMS 0500333).

(C)2009 American Mathematical Society Reverts to public domain 28 years from publication 
However, $H_{\alpha}$ and $H_{\beta}$ are intuitively the same type of submodules. The rigidity phenomenon indicates that, for the purpose of classifying submodules, one needs a more flexible equivalence relation. Congruence of submodules was defined in Ya2. While it is still far from a complete classification of all submodules, the congruence relation is able to make good progress in this quest, as we will see in the next section.

\section{Core operator and Congruence}

In this paper $K(\lambda, z)=\left(1-\overline{\lambda_{1}} z_{1}\right)^{-1}\left(1-\overline{\lambda_{2}} z_{2}\right)^{-1}$ is the reproducing kernel for $H^{2}\left(D^{2}\right)$. The reproducing kernel for a submodule $M$ is denoted by $K^{M}(\lambda, z)$. The core function $G^{M}(\lambda, z)$ for $M$ is

$$
G^{M}(\lambda, z):=\frac{K^{M}(\lambda, z)}{K(\lambda, z)}=\left(1-\overline{\lambda_{1}} z_{1}\right)\left(1-\overline{\lambda_{2}} z_{2}\right) K^{M}(\lambda, z),
$$

and the core operator $C^{M}$ (or simply $C$ ) on $H^{2}\left(D^{2}\right)$ is given by

$$
C^{M}(f)(z):=\int_{T^{2}} G^{M}(\lambda, z) f(\lambda) d m(\lambda), \quad z \in D^{2},
$$

where $d m(\lambda)$ is the normalized Lebesgue measure on $T^{2}$. The core operator is introduced in GY]. More studies can be found in Ya1 and Ya2. A basic fact is that on every submodule $M, C$ is a bounded self-adjoint operator with $\|C\|=1$. Moreover, it is not hard to check that $C=0$ on $M^{\perp}$, so $C$ will be restricted to $M$ in our study.

For a submodule $M$ we let $\left(R_{1}, R_{2}\right)$ be the pair of multiplications by $z_{1}$ and $z_{2}$ on $M$. Clearly, $\left(R_{1}, R_{2}\right)$ is a pair of commuting isometries on $M$. One relation between the core operator and the pair $\left(R_{1}, R_{2}\right)$ is the identity

$$
C=1-R_{1} R_{1}^{*}-R_{2} R_{2}^{*}+R_{1} R_{2} R_{1}^{*} R_{2}^{*} .
$$

A submodule $M$ is said to be $c$-compact (c-finite) if its core operator $C$ is compact (or, respectively, of finite rank). There are many $c$-finite submodules, and as indicated in [Ya2, almost all known examples of submodules are $c$-compact (in fact Hilbert-Schmidt). Two submodules $M$ and $N$ are said to be congruent if $C^{M}$ and $C^{N}$ are congruent, e.g., there is a bounded invertible linear operator $J$ from $N$ to $M$ such that $C^{M}=J C^{N} J^{*}$.

Example 2. Now consider the action $L$ of $\operatorname{Aut}\left(D^{2}\right)$ on $H^{2}\left(D^{2}\right)$ defined by

$$
\left(L_{x} f\right)(z)=f(x(z)), \quad x \in \operatorname{Aut}\left(D^{2}\right),
$$

where $\operatorname{Aut}\left(D^{2}\right)$ is the group of bi-holomorphic self-maps on $D^{2}$. One sees that $L_{x}$ is bounded invertible and $L_{x}(M)$ is a submodule. Moreover, by Ya2,

$$
C^{L_{x}(M)}=L_{x} C^{M} L_{x}^{*} .
$$

Hence $M$ and $L_{x}(M)$ are congruent. In particular, $H_{\alpha}$ and $H_{\beta}$ in Example 1 are congruent.

An invertible symmetric matrix $A$ is said to have signature $(p, q)$ if there is a nondegenerate matrix $T$ such that $T A T^{*}$ is a diagonal matrix with $p 1$ s and $q-1$ s. Since signature is a complete invariant of congruence relation for invertible self-adjoint matrices, it follows easily that two $c$-finite submodules $M$ and $N$ are congruent if and only if $C^{M}$ and $C^{N}$, when restricted to the orthogonal complement of their kernels, have the same signature (cf. YYa2]). The main purpose of this paper 
is to improve on this fact and show that the rank itself is a complete invariant for congruent $c$-finite submodules.

The following lemma from [Ya2] is needed.

Lemma 1.1. $C^{2}$ is unitarily equivalent to the diagonal block matrix

$$
\left(\begin{array}{ll}
{\left[R_{1}^{*}, R_{1}\right]\left[R_{2}^{*}, R_{2}\right]\left[R_{1}^{*}, R_{1}\right]} & 0 \\
0 & {\left[R_{2}^{*}, R_{1}\right]^{*}\left[R_{2}^{*}, R_{1}\right]}
\end{array}\right) .
$$

For an operator $A$ with an eigenvalue $\lambda$, we let $E_{\lambda}(A)$ denote the corresponding eigenspace. It is shown in $[\mathrm{GY}]$ that

$$
E_{1}(C)=M \ominus\left(z_{1} M+z_{2} M\right), \quad E_{-1}(C)=\left(z_{1} M \cap z_{2} M\right) \ominus z_{1} z_{2} M .
$$

The next lemma is concerned with eigenvalues in the open interval $(-1,1)$.

Lemma 1.2. Let $M$ be a submodule, and let $\lambda$ be a nonzero eigenvalue of $C$ in $(-1,1)$. Then $-\lambda$ is also an eigenvalue, and moreover $\operatorname{dim} E_{\lambda}(C)=\operatorname{dim} E_{-\lambda}(C)$.

Proof. Assume $\lambda$ is a nonzero eigenvalue of $C$ in $(-1,1)$. For any nontrivial $f \in$ $E_{\lambda}(C)$, we have

It follows from (1-1) that

$$
R_{2}^{*} C f=\lambda R_{2}^{*} f
$$

$$
\begin{aligned}
\lambda R_{2}^{*} f & =R_{2}^{*}\left(I-R_{1} R_{1}^{*}-R_{2} R_{2}^{*}+R_{1} R_{2} R_{1}^{*} R_{2}^{*}\right) f \\
& \left.=R_{2}^{*}-R_{2}^{*} R_{1} R_{1}^{*}-R_{2}^{*}+R_{1} R_{1}^{*} R_{2}^{*}\right) f \\
& =-\left(R_{2}^{*} R_{1}-R_{1} R_{2}^{*}\right) R_{1}^{*} f .
\end{aligned}
$$

Parallelly, we have

$$
\lambda R_{1}^{*} f=-\left(R_{1}^{*} R_{2}-R_{2} R_{1}^{*}\right) R_{2}^{*} f .
$$

We first observe that $R_{2}^{*} f \neq 0$. Since if $R_{2}^{*} f=0$, by (1-3), $R_{1}^{*} f$ is also equal to 0 . This means that $f \in M \ominus\left(z_{1} M+z_{2} M\right)$, which contradicts the fact that $\lambda \neq 1$.

Putting (1-3) into (1-2), we have

$$
\left[R_{2}^{*}, R_{1}\right]\left[R_{1}^{*}, R_{2}\right] R_{2}^{*} f=\lambda^{2} R_{2}^{*} f .
$$

In conclusion, $R_{2}^{*}: E_{\lambda}(C) \longrightarrow E_{\lambda^{2}}\left(\left[R_{2}^{*}, R_{1}\right]\left[R_{1}^{*}, R_{2}\right]\right)$ is a well-defined injective map. In particular,

$$
\operatorname{dim} E_{\lambda}(C) \leq \operatorname{dim} E_{\lambda^{2}}\left(\left[R_{2}^{*}, R_{1}\right]\left[R_{1}^{*}, R_{2}\right]\right) .
$$

On the other hand, if we multiply the equation $C f=\lambda f$ by $\left[R_{1}^{*}, R_{1}\right]$ and using (1-1), we have

$$
\begin{aligned}
& \lambda\left[R_{1}^{*}, R_{1}\right] f=\left[R_{1}^{*}, R_{1}\right]\left(I-R_{1} R_{1}^{*}-R_{2} R_{2}^{*}+R_{1} R_{2} R_{1}^{*} R_{2}^{*}\right) f \\
& =\left[R_{1}^{*}, R_{1}\right]\left(I-R_{2} R_{2}^{*}\right) f+\left[R_{1}^{*}, R_{1}\right]\left(-R_{1} R_{1}^{*}+R_{1} R_{2} R_{1}^{*} R_{2}^{*}\right) f \\
& =\left[R_{1}^{*}, R_{1}\right]\left[R_{2}^{*}, R_{2}\right] f .
\end{aligned}
$$

Parallelly, multiplying the equation $C f=\lambda f$ by $\left[R_{2}^{*}, R_{2}\right]$ and using (1-1), we have

$$
\lambda\left[R_{2}^{*}, R_{2}\right] f=\left[R_{2}^{*}, R_{2}\right]\left[R_{1}^{*}, R_{1}\right] f .
$$

First we observe that $\left[R_{1}^{*}, R_{1}\right] f \neq 0$. Since if $\left[R_{1}^{*}, R_{1}\right] f=0$, then by (1-7), $\left[R_{2}^{*}, R_{2}\right] f$ is also 0 . These imply that $f \in z_{1} M \cap z_{2} M$. Since it is easy to see that $z_{1} z_{2} M \subset \operatorname{ker} C, f \in z_{1} M \cap z_{2} \ominus z_{1} z_{2} M=E_{-1}(C)$, and this contradicts the fact that $\lambda \neq-1$. 
Now combining (1-6) and (1-7), we have

$$
\left[R_{1}^{*}, R_{1}\right]\left[R_{2}^{*}, R_{2}\right]\left[R_{1}^{*}, R_{1}\right] f=\lambda^{2}\left[R_{1}^{*}, R_{1}\right] f .
$$

Since $\left[R_{1}^{*}, R_{1}\right]=\left[R_{1}^{*}, R_{1}\right]^{2}$, these observations show that

$$
\left[R_{1}^{*}, R_{1}\right]: E_{\lambda}(C) \longrightarrow E_{\lambda^{2}}\left(\left[R_{1}^{*}, R_{1}\right]\left[R_{2}^{*}, R_{2}\right]\left[R_{1}^{*}, R_{1}\right]\right)
$$

is a well-defined injective map. In particular,

$$
\operatorname{dim} E_{\lambda}(C) \leq \operatorname{dim} E_{\lambda^{2}}\left(\left[R_{2}^{*}, R_{1}\right]\left[R_{1}^{*}, R_{2}\right]\right) .
$$

It now follows from Lemma 1.1 that

$$
\operatorname{dim} E_{\lambda^{2}}\left((C)^{2}\right) \geq 2 \operatorname{dim} E_{\lambda}(C),
$$

which implies that

$$
\operatorname{dim} E_{-\lambda}(C) \geq \operatorname{dim} E_{\lambda}(C) .
$$

The same line of arguments starting with $-\lambda$ will prove the inequality in the other direction, and the proof is complete.

If $C$ is compact, then $\overline{\operatorname{ran}(C)}$ can be decomposed as

$$
\overline{\operatorname{ran}(C)}=E_{1} \oplus\left(\bigoplus_{0<\lambda_{j}<1} E_{\lambda_{j}}\right) \oplus E_{-1} \oplus\left(\bigoplus_{-1<\lambda_{j}<0} E_{\lambda_{j}}\right) .
$$

For simplicity, we let $d_{1}=\operatorname{dim} E_{1}, d_{-1}=\operatorname{dim} E_{-1}$, and

$$
D=\bigoplus_{0<\lambda_{j}<1} \lambda_{j} P_{j},
$$

where $P_{j}$ is the orthogonal projection from $M$ onto $E_{\lambda_{j}}$. Then Lemma 1.2 indicates that $C$ is unitarily equivalent to the diagonal block matrix

$$
\left(\begin{array}{llll}
I_{d_{1}} & 0 & 0 & 0 \\
0 & D & 0 & 0 \\
0 & 0 & -I_{d_{-1}} & 0 \\
0 & 0 & 0 & -D
\end{array}\right) .
$$

Theorem 1.3. Two c-finite submodules $M$ and $N$ are congruent if and only if $C^{M}$ and $C^{N}$ have the same rank.

Proof. If $M$ and $N$ are congruent c-finite submodules, then $C^{M}$ and $C^{N}$ have the same signature by $\mathrm{Ya2}$, and hence $C^{M}$ and $C^{N}$ have the same rank.

For the sufficiency, it is shown in GY] that if $C$ is trace class, then $\operatorname{tr} C=1$. In view of (1-10), this fact implies $d_{1}=d_{-1}+1$. So if $C^{M}$ and $C^{N}$ have the same rank, then by (1-10) they have the same signature. Hence $M$ and $N$ are congruent.

Example 3. It is known that $\operatorname{rank}(C)=1$ if and only if $M=\phi H^{2}\left(D^{2}\right)$ for some inner function $\phi$ (cf. GY] $)$. So by Theorem 1.3, $M$ is congruent to $H^{2}\left(D^{2}\right)$ if and only if $M$ is of the form $\phi H^{2}\left(D^{2}\right)$.

It follows from (1-10) and the fact that $d_{1}=d_{-1}+1$ that for a $c$-finite submodule, the rank of $C$ is always an odd number. So next in line is the case $\operatorname{rank} C=3$. 
Example 4. If $q_{1}\left(z_{1}\right), q_{2}\left(z_{2}\right)$ are two nontrivial one-variable inner functions over the unit disk $D$, then

$$
M=q_{1}\left(z_{1}\right) H^{2}\left(D^{2}\right)+q_{2}\left(z_{2}\right) H^{2}\left(D^{2}\right)
$$

is a submodule with interesting properties (cf. Izuchi, Nakazi and Seto [INS]). It is not difficult to compute that $\operatorname{rank} C=3$.

Another type of submodule $M$ with $\operatorname{rankC}=3$ is of the form

$$
M=\phi H^{2}\left(D^{2}\right) \oplus \frac{\phi H(z)}{w-G(z)} H^{2}(z),
$$

where $\phi$ is an inner function, $G(z)$ and $H(z)$ are in the unit ball of $H^{\infty}(D)$ that satisfy some conditions, and $H^{2}(z)$ is $H^{2}(D)$ in the variable $z$ (cf. K. J. Izuchi and K. H. Izuchi [I] ).

Question. Is it possible to characterize all submodules $M$ with $\operatorname{rankC}=3$ ?

\section{REFERENCES}

[CG] X. Chen and K. Guo, Analytic Hilbert Modules, Chapman \& Hall/CRC, Boca Raton, FL, 2003. MR1988884 (2004d:47024)

[DPSY] R. Douglas, V. Paulsen, C.-H. Sah and K. Yan, Algebraic reduction and rigidity for Hilbert modules, Amer. J. Math. 117 (1995), No. 1, 75-92. MR1314458 (95k:46113)

[GY] K. Guo and R. Yang, The core function of submodules over the bidisk, Indiana Univ. Math. J. 53 (2004), 205-222. MR2048190(2005m:46048)

[II] K. J. Izuchi and K. H. Izuchi, Rank one commutators on invariant subspaces of the Hardy space on the bidisk, J. Math. Anal. Appl. 316 (2006), 1-8. MR2201744 (2006k:47012)

[INS] K. Izuchi, T. Nakazi and M. Seto, Backward shift invariant subspaces in the bidisk (II), J. Oper. Theory 51 (2004), No. 2, 361-376. MR2074186 (2005c:47008)

[Ru] W. Rudin, Function Theory in Polydisks, W. A. Benjamin, Inc., New York-Amsterdam, 1969. MR0255841(41:501)

[Ya1] R. Yang, On two-variable Jordan blocks, Acta Sci. Math. (Szeged) 69 (2003), No. 3-4, 739-754. MR2034205 (2004j:47011)

[Ya2] R. Yang, The core operator and congruent submodules, J. Funct. Anal. 228 (2005), No. 2, 469-489. MR2175415(2006e:47015)

Department of Mathematics and Statistics, The State University of New York at Albany, Albany, New York 12222

E-mail address: ryang@@math.albany.edu 